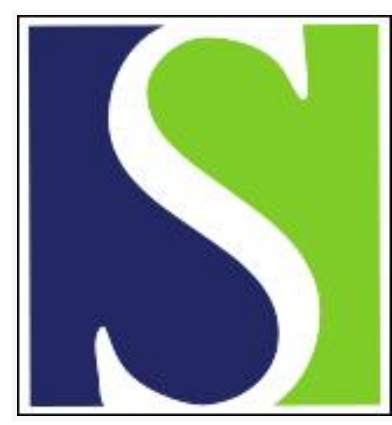

Scand J Work Environ Health 1990;16(4):289-292

https://doi.org/10.5271/sjweh.1782

Issue date: 01 Aug 1990

Liver injury in workers exposed to dimethylformamide. by Fleming LE, Shalat SL, Redlich CA

Affiliation: Department of Epidemiology and Public Health, University of Miami School of Medicine, Florida.

This article in PubMed: www.ncbi.nlm.nih.gov/pubmed/2389137 


\title{
Liver injury in workers exposed to dimethylformamide
}

\author{
by Lora E Fleming, MD, MPH, ${ }^{1}$ Stuart L Shalat, $S c D,{ }^{2}$ Carrie A Redlich, MD, $\mathrm{MPH}^{3}$
}

\begin{abstract}
FLEMING LE, SHALAT SL, REDLICH CA. Liver injury in workers exposed to dimethylformamide. Scand J Work Environ Health 1990;16:289-92. An apparent epidemic cluster of toxic liver disease was reexamined among workers exposed to the solvent dimethylformamide. A demographically similar but unexposed group from a preemployment population was used for comparison. Analysis, after data transformation of the liver transaminases, revealed significant differences between the two populations with respect to the serum glutamic pyruvic transaminase and the ratio of serum glutamic oxaloacetic transaminase to serum glutamic pyruvic transaminase. Thus a value of the ratio $<1$ may be suggestive of toxic liver disease. Medical surveillance of the working population for 14 months revealed no further cases of toxic liver disease. Dimethylformamide was almost certainly the causative agent of the original epidemic. The use of preemployment populations as a source of unexposed subjects in the analysis of occupational clusters is recommended, especially in the scenario of relatively acute, and highly prevalent, occupational diseases.
\end{abstract}

Key terms: cluster analysis, occupational clusters, preemployment screening.

The evaluation of an occupational disease often involves cluster analysis or aggregate analysis or both in that small groups of workers in a given industrial location are reported to have an increased prevalence of some disease which may or may not be related to their occupational exposures. However, these analyses can be difficult. Small numbers, inadequate reference populations, multiple exposure agents, and population factors, not to mention the possibility of chance alone leading to the apparent increase, are all problems plaguing these analyses $(1-6)$. Nevertheless, we feel that various aspects of cluster and aggregate analyses, previously applied to cancer and infectious disease problems, are relevant to the investigation of occupational and environmental diseases.

In 1986, we discovered a cluster of toxic liver disease among workers exposed to the solvent dimethylformamide (7). Thirty-five out of the 45 exposed production workers had abnormalities of their liver transaminases [serum glutamic oxaloacetic transaminase (SGOT) (aspartate aminotransferase) and serum glutamic pyruvic transaminase (SGPT) (alanine aminotransferase)], while only one out of the 12 unexposed nonproduction workers had such abnormalities. Specifically, the SGOT and/or SGPT level was significantly elevated and the SGOT:SGPT ratio was $<1$ for all

1 Department of Epidemiology and Public Health, University of Miami School of Medicine, Miami, Florida, United States.

2 Department of Occupational Medicine, Yale Medical School, New Haven, Connecticut, United States.

3 Department of Occupational Medicine, University of Washington, Seattle, Washington, United States.

Reprints requests to: Dr LE Fleming, Department of Epidemiology and Public Health (R-669), University of Miami School of Medicine, PO Box 016069, Miami, FL 33101, USA. but one of the exposed population. At the same time, other indices of liver abnormality (such as bilirubin, alkaline phosphatase, and viral serologies) were normal in the entire study population. Finally, liver biopsies (including special fat stains and electron microscopy) obtained from seven exposed individuals showed evidence of microvesicular fat and hepatocellular unrest, without significant inflammatory infiltrate. It was felt that the overall clinical and pathological picture was consistent with an epidemic of liver disease of a toxic origin $(7,8)$.

Concerns about this preliminary study included the following: the small number of demographically different unexposed subjects, the inclusion of the three index cases, the possibility of some unidentified "population" factor (such as increased prevalence of obesity, diabetes, alcohol abuse, non A non B viral hepatitis, previous occupational exposures, or merely a different distribution of liver transaminases among the population from which the exposed group of workers came), the statistical distribution of the liver transaminases, and the assertion that dimethylformamide was the causative agent. We undertook the following analysis in order to address these concerns and to establish that this cluster represented a real epidemic of occupational liver disease.

\section{Subjects and methods}

Due to our role as the medical director of the company in which the epidemic of toxic liver disease took place, over the past 14 months we were able to collect a large case file of individuals undergoing preemployment medical screening. This screening involved, among other things, a complete liver profile (ie, the liver transaminases SGOT and SGPT, alkaline phos- 
phatase and bilirubin). Using this information, we were able to assemble a population of men who were unexposed and demographically similar as the unexposed population in our reanalysis. It should be noted that preemployment data (including height and weight) did not exist for the exposed individuals.

To evaluate the actual cluster of exposed workers, we elected to exclude from our analysis the three index cases since they could bias our results. We decided to look only at the population of both the exposed and the unexposed subjects who fulfilled the following criteria: male, Hispanic (by last name), and only in exposed jobs (ie, production, laminator, quality control). This procedure created an exposed population of 41 individuals from the original 45 , and an unexposed population of 111 individuals from the preemployment screening group of 136 . We evaluated only the initial liver transaminase values of all these individuals.

We reanalyzed the statistical distribution of the liver transaminases and their ratio by performing a $\log$ 10 transformation. This transformation not only gave a more normal distribution, but was also in accordance with existing literature on the analysis of liver transaminase values $(9,10)$. We then compared the exposed and unexposed populations by performing t-tests, an analysis of possible confounding variables, and, finally, a multivariate regression analysis (including analysis of covariance) using the various relevant variables available. These variables consisted of exposure status, age, and liver transaminases with their ratio (ie, SGOT:SGPT ratio); other variables such as race, sex, and job category were the same between the exposed and unexposed groups. All the statistical analyses were performed with the use of the SAS (statistical analysis system) program, version 6.02 .

We were concerned about the possible confounding effects of age. It was possible that the duration of employment could be collinear with age. The original investigation had shown that the liver transaminases actually decreased with duration of employment (7). In addition, there was evidence from the t-test that age was significantly different between the two exposure groups $(P=0.006)$. A regression analysis looking at age alone in each exposure group separately revealed no significant effect of age on any of the transformed liver transaminases or their ratio. Therefore, age did not appear to be an effect modifier.

Finally, several engineering and industrial hygiene changes, with worker education in Spanish and Eng- lish, were implemented in the workplace through the efforts of the union and management. In addition a dramatic reduction occurred in the actual amount of dimethylformamide used in production. Finally, medical surveillance in the form of a liver-profile blood screen, carried out every three months, and a complete annual medical evaluation had been implemented over the past 14 months.

\section{Results}

First, we examined basic demographic information to make comparisons between the exposed $(\mathrm{N}=41)$ and unexposed $(\mathrm{N}=111)$ subjects. The exposed group was slightly older on the average [mean 33.5 (median 29) years] than the unexposed group [mean 28.2 (median 25) years] at the time of the initial screening. A t-test indicated that the age difference between the two groups was statistically significant $(P=0.006)$.

We then examined the liver transaminases in each of the two groups and found that the means and medians of the liver transaminases and their ratio were very different within each exposure group; that is, there was evidence of a skewed distribution. We performed $\log 10$ transformations of the liver transaminase data, and these transformations markedly normalized the distributions in that they led to an increased correspondence between the means and medians within each exposure group. (See table 1 and figure 1.)

We then looked at t-test analyses that compared the two groups after the log 10 transformations of the data. In some contrast to the original investigation, we found that the SGPT and the SGOT:SGPT ratio were significantly different between the two groups ( $\mathrm{P}<0.0001$ for both), while the SGOT difference was no longer statistically significant.

Since we were still concerned about a possible age effect on the liver transaminase distributions and the confounding effect of age in general, we elected to keep age as a variable in the final analysis. We examined, by multivariate regression analysis, models which included the variables of exposure (a dichotomous variable: exposed versus unexposed) and age (a continuous variable) for each of the transformed liver transaminases and their ratio. The analysis showed that there were significant effects of exposure (with age) on the liver transaminases and their ratio (with $\mathrm{P}<0.0001$ in the F-test for the SGPT and the ratio). In other words, those workers who were in the exposed

Table 1. Untransformed and transformed (log 10) liver transaminase levels [serum glutamic oxaloacetic transaminase (SGOT) and serum glutamic pyruvic transaminase (SGPT)] and their ratio (SGOT:SGPT).

\begin{tabular}{|c|c|c|c|c|c|c|c|c|c|c|c|c|c|}
\hline \multirow{3}{*}{ Group } & \multirow{3}{*}{$\mathrm{N}$} & \multicolumn{4}{|c|}{$\begin{array}{l}\text { SGOT (aspartate } \\
\text { aminotransferase) }\end{array}$} & \multicolumn{4}{|c|}{$\begin{array}{l}\text { SGPT (alanine } \\
\text { aminotransferase) }\end{array}$} & \multicolumn{4}{|c|}{ SGOT: SGPT } \\
\hline & & \multicolumn{2}{|c|}{ Untransformed } & \multicolumn{2}{|c|}{ Transformed } & \multicolumn{2}{|c|}{ Untransformed } & \multicolumn{2}{|c|}{ Transformed } & \multicolumn{2}{|c|}{ Untransformed } & \multicolumn{2}{|c|}{ Transformed } \\
\hline & & Mean & Median & Mean & Median & Mean & Median & Mean & Median & Mean & Median & Mean & Median \\
\hline $\begin{array}{l}\text { Exposed } \\
\text { Unexposed }\end{array}$ & $\begin{array}{r}41 \\
111\end{array}$ & $\begin{array}{l}64 \\
42\end{array}$ & $\begin{array}{l}35 \\
30\end{array}$ & $\begin{array}{l}1.62 \\
1.52\end{array}$ & $\begin{array}{l}1.54 \\
1.48\end{array}$ & $\begin{array}{l}94 \\
46\end{array}$ & $\begin{array}{l}49 \\
28\end{array}$ & $\begin{array}{l}1.77 \\
1.49\end{array}$ & $\begin{array}{l}1.69 \\
1.45\end{array}$ & $\begin{array}{l}0.73 \\
1.16\end{array}$ & $\begin{array}{l}0.71 \\
1.07\end{array}$ & $\begin{array}{l}-0.15 \\
0.025\end{array}$ & $\begin{array}{c}-0.15 \\
0.029\end{array}$ \\
\hline
\end{tabular}



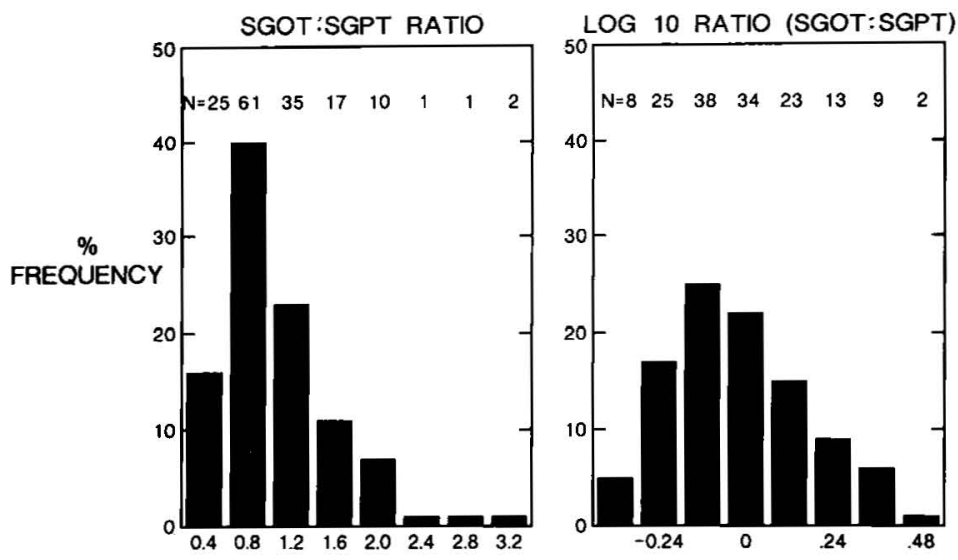

Figure 1. Distribution of the untransformed and transformed $(\log 10)$ ratios of serum glutamic oxaloacetic transaminase (SGOT) to serum glutamic pyruvic transaminase (SGPT) (exposed and unexposed workers combined).

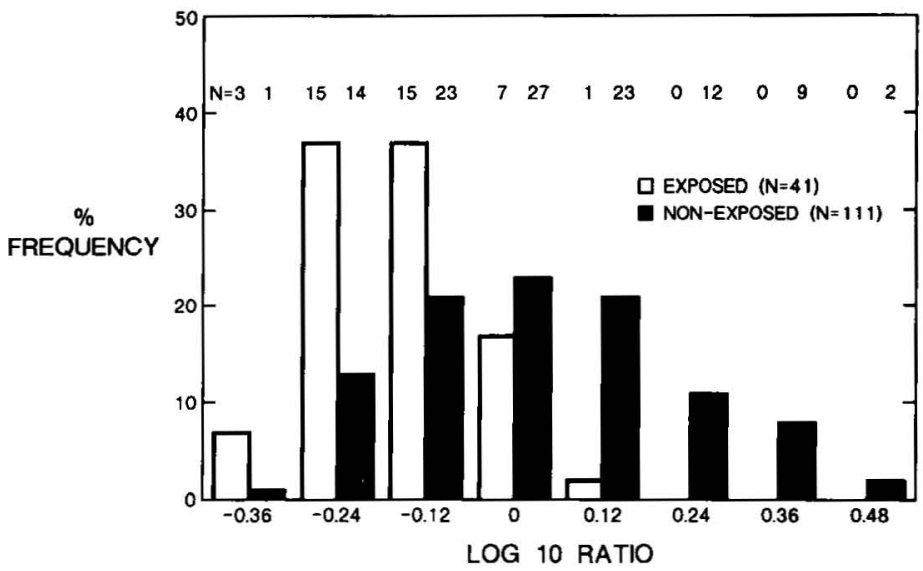

Figure 2. Distribution of the transformed $(\log 10)$ ratios (serum glutamic oxaloacetic transaminase: serum glutamic pyruvic transaminase) of the exposed and unexposed subjects separately. (Non-exposed $=$ unexposed)

group were more likely to have an increased SGPT level and a SGOT:SGPT ratio of $<1$ than the unexposed group. (See figure 2.) Again, the SGOT level was not found to be statistically different between the two groups. Of note, we did examine the possibility of covariance between the two variables exposure and age in our model and found it to be an appropriate model with parallelism present and a normal distribution of the residuals.

Finally, the 14-month medical surveillance system, with the engineering and industrial hygiene controls already described, revealed no definite new cases of liver disease in this workplace.

\section{Discussion}

We reexamined an apparent epidemic cluster of toxic liver disease among workers exposed to the solvent dimethylformamide. First we provided a new, larger, and demographically similar unexposed group for comparison. This population consisted of 111 Hispanic men applying for exposed jobs. We also excluded the original three index cases from the analysis. We thus created two very similar groups in terms of demographic and, presumably, medical characteristics.
We then performed log 10 transformations of the data in order to gain more normalized distributions and thus a more statistically valid analysis of the data. A multivariate regression analysis, after analysis for possible confounding and covariance, revealed a significant effect of exposure on the liver transaminase status. Those who were exposed had significantly elevated SGPT levels and were more likely to have an SGOT:SGPT ratio of $<1$.

From this analysis, we conclude the following. First, the elevation of liver transaminases, in particular the SGPT level, was not attributable to a population factor since we used as our comparison a demographically similar population. Since $20 \%$ of the unexposed population (as opposed to $59 \%$ of the exposed) did have one or more liver transaminase values greater than the laboratory normal for the liver transaminases, it is possible that there was an increased rate of such factors as obesity, diabetes, non A non B viral hepatitis, alcohol use, other occupational exposures, acquired immunodeficiency disease, or intravenous drug use in this general population. However, it should be noted that there appeared to be a normal distribution of liver transaminases within this population, with data trans- 
formation, which suggests that there may be some slightly different population liver transaminase distribution rather than an infectious or toxic etiology. In terms of our own analysis, this possibility is irrelevant since it did not prevent us from showing a statistically different increase in the liver transaminases and their ratio among the exposed workers when they were compared with an unexposed population.

As with the preliminary investigation, we affirm that an SGOT:SGPT ratio of $<1$, especially when each of the liver transaminases is $<300 \mathrm{IU} / \mathrm{l}$, may be suggestive of the diagnosis of toxic liver disease (7). This can be a useful criterion for worker populations in which the liver transaminases are often not so elevated as to suggest acute viral hepatitis, but their increase does suggest the possibility of alcoholic hepatitis. In addition, an elevated level of gamma glutamyl transpeptidase, while extremely sensitive to any liver insult, does not distinguish between alcoholic and toxic hepatitis (11, 12). However, past work has shown that with elevated liver transaminases of $<300$ and with SGOT:SGPT ratios of $>2$, there is a strong possibility of an alcoholic etiology (13). Of note, 40 of our 41 exposed subjects had a SGOT:SGPT ratio of $<1$, and all the subjects' liver transaminases were below 300 IU/1. None of our subjects were known diabetics, and, unfortunately, height and weight were not available for the exposed individuals.

As was mentioned, during the 14 months of medical surveillance, after significant reduction in and protection from the use of dimethylformamide, there have been no new cases of toxic liver disease - despite the new exposure of 138 new employees in this workplace and the introduction of new solvents such as trichloroethylene and methylene chloride. Thus we are almost certain that dimethylformamide (or dimethylformamide in synergism with some other agent or agents) was the causative agent of the original epidemic of toxic liver disease. This possibility is further supported by preexisting evidence in the literature of dimethylformamide hepatotoxicity in both humans and animals (14). Therefore, we recommend that this chemical, with its extensive use and significant skin absorption, be monitored closely in the future, especially since recent investigations suggest that, in addition to its hepatotoxic effects, dimethylformamide may also be a testicular carcinogen in humans $(15,16)$.

We recommend the use of preemployment screening as a useful source of unexposed comparison populations in the analysis of occupational clusters. We believe that this technique would be especially useful in clusters of fairly acute and highly prevalent diseases (such as respiratory morbidity in the case of indoor air pollution or acute toxic scenarios such as we have described). In addition, it is also useful in workplaces in which there is a relatively high turnover of employees or a relatively large work force since it leads rapidly to large numbers of preemployment individuals for comparison groups in occupational cluster analysis.
Finally, we hope that the work which has already been done in infectious disease and cancer cluster analysis will be applied more extensively in the future to occupational and environmental settings.

\section{Acknowledgments}

We would like to acknowledge M Cullen, MD, for his . insightful comments, C Reily, MD, for her expert assistance, M Ortiz, BS, for his support, and the workers, whose contribution has been anonymous but essential to this study. We also thank Ms M Moran, the ILGWU, and Mr S Jacobs for their technical support.

Dr Fleming was supported by a Dana Foundation grant during the course of the research presented in this paper.

\section{References}

1. Mantel N. The detection of disease clustering and a generalized regression approach. Cancer Res 1967;27: $209-19$.

2. Smith PG. Spatial and temporal clustering. In: Schottenfeld D, Fraumeni JF, ed. Cancer epidemiology and prevention. Philadelphia, PA: WB Saunders Company, 1982:728-38.

3. Rothman K. Clustering of disease. Am J Public Health 1987;77:13-7.

4. Schulte PA, Ehrenberg RA, Singal M. Investigation of occupational cancer cluster: theory and practice. Am J Public Health 1987;77:52-6.

5. Frumkin H, Kantrowitz W. Cancer clusters in the workplace: an approach to investigation. J Occup Med 1987; 29:949-52.

6. Enterline PE. Evaluating cancer clusters. Am Ind Hyg Assoc J 1985;46:B10-B13.

7. Redlich CA, Beckett WS, Sparer J, et al. Liver disease associated with occupational exposure to the solvent Dimethylformamide. Ann Intern Med 1988;108:680-6.

8. Zimmerman HJ. Hepatotoxicity: the adverse effects of drugs and other chemicals on the liver. New York, NY: Appleton-Century-Croft, 1978.

9. Coulton T. Statistics in medicine. Boston, MA: Little Brown \& Co, 1974:87-9.

10. Prince AM, Gershon RK. The use of serum enzyme determination to detect anicteric hepatitis. Transfusion 1965;5:120.

11. Dossing M. Noninvasive assessment of microsomal enzyme activity in occupational medicine: present state of knowledge and future perspectives. Int Arch Occup Environ Health 1984;53:205-18.

12. Tamburro CH, Liss GM. Tests for hepatotoxicity: usefulness in screening workers. J Occup Med 1986;28: $1034-44$.

13. Cohen JA, Kaplan MM. The SGOT/SGPT ratio an indicator of alcoholic liver disease. Dig Dis Sci 1979;24: 835-8.

14. Kennedy GL Jr. Biological effects of acetamide, formamide, and their monomethyl and diethyl derivatives. CRC Crit Rev Toxicol 1986;17:129-82.

15. Ducatman AM, Conwill DE, Crawl J. Germcell tumors of the testicle among aircraft repairmen. J Urol 1986; 136:834-6.

16. Levin SM, Baker DB, Landrigan PJ, et al. Testicular cancer in leather tanners exposed to Dimethylformamide. Lancet 1987;2:1153.

Received for publication: 18 December 1989 